\title{
22. AMINO ACIDS, AMINO SUGARS, AND AMMONIA IN SEDIMENTS FROM THE CARIACO TRENCH
}

\author{
P. E. Hare, Geophysical Laboratory, 2801 Upton Street, NW, Washington, D.C.
}

\section{INTRODUCTION}

Bulk sediment samples from 1 meter, 80 meters, and 110 meters below sea floor Site 147 were freeze dried, hydrolyzed with hydrochloric acid, demineralized, and analyzed for amino acids, amino sugars, and ammonia.

\section{SAMPLE PREPARATION}

One gram aliquots of the freeze-dried bulk sediment samples were treated with concentrated $\mathrm{HC} 1$ to react with any carbonates present. Additional acid was added to obtain a $6 \mathrm{~N} \mathrm{HC1}$ solution after which the samples were sealed under nitrogen and heated at $110^{\circ} \mathrm{C}$ for twenty-two hours to liberate bound amino acids. After hydrolysis the insoluble residue was centrifuged down and the supernatant liquid transferred to a polyethylene tube. Concentrated hydrofluoric acid was added drop by drop to precipitate calcium as the fluoride. The $\mathrm{CaF}_{2}$ was centrifuged down and the supernatant liquid transferred to another polyethylene tube and taken to dryness in a vacuum centrifuge. The dried residue was dissolved in $0.01 \mathrm{~N} \mathrm{HC} 1$ and the $\mathrm{pH}$ adjusted to 10 with $2 \mathrm{~N} \mathrm{NaOH}$. This step precipitated such interfering cations as iron. After centrifugation the clear solution was acidified with $\mathrm{HC} 1$ to $\mathrm{pH} 2.0$. Aliquots were applied directly to the ion-exchange column of an ultra sensitive amino acid analyzer (Hare, 1971).

Experiments with controls and standard amino acid mixtures show this procedure results in negligible losses of ammonia and amino acids. Recovery is approximately 90 per cent with no indication of any selective losses.

The classical procedure in extracting amino acids followed in most previous studies involves the use of cationexchange resin. The amino acids are eluted off the resin column with ammonium hydroxide. This procedure is satisfactory for the neutral and acidic amino acids but is not quantitative for the basic amino acids and, of course, is useless for the determination of ammonia simultaneously with the analysis of the amino acids.

The procedure described here involves a single evaporation step. The precipitation steps are carried out within a few minutes in the cold or even at room temperature. Comparing results with those obtained from ion-exchange desalting show this procedure is more satisfactory and much faster. The presence of $\mathrm{NaCl}$ in the final sample is not a serious problem in the ion-exchange method of analysis, since the buffers used are $0.2 \mathrm{~N} \mathrm{Na}^{+}$. Concentrations of even somewhat more than $1 \mathrm{~N} \mathrm{NaCl}$ do not adversely affect the resulting chromatogram. However,
$\mathrm{NaCl}$ can be removed easily by bubbling $\mathrm{HCl}$ through the solution at $0^{\circ} \mathrm{C}$. The supernatant liquid is then evaporated to dryness and the salt-free residue made up to volume for analysis. This may be necessary if derivatives are to be made for gas-liquid chromatographic methods.

\section{RESULTS AND DISCUSSION}

The amino acid, amino sugar, and ammonia concentrations are listed in Table 1. All of the common protein amino acids were found. In addition, several other amino acids and ninhydrin positive compounds were found. Besides those listed in the table, minor peaks (probably less than 5 nanomoles per gram) were found for urea, taurine, alpha amino butyric acid, diamino pimelic acid, hydroxylysine, ethanolamine, methylamine, and ethylamine. A trace of cystine was found in the near-surface sample but not in the others.

Concentrations of most of the amino acids decrease by factors of up to ten as the depth of the sample increases. For example, less than 10 per cent of the aspartic acid present in the near-surface sediment sample is present in the sample from 110 meters. Most of the protein amino acids show similar concentration decreases with depth of sample. The nonprotein amino acids and amino sugars do not show this relationship. Some are diagenetic products from protein amino acids and are possibly in a steady state concentration as breakdown of the parents proceeds.

In contrast to the amino acid concentration, the ammonia concentration actually increases with the depth of the sample. Ammonia accounts by far for the largest fraction of the ninhydrin positive compounds. The ratio of ammonia to amino acids is approximately 2 to 1 in the near-surface sample, 6 to 1 in the 80 -meter sample, and 10 to 1 in the 110 -meter sample. This data suggest that much of the ammonia may be the result of deamination of the amino acids.

Low molecular weight amines, products of decarboxylation reactions of amino acids, are present in small quantities in these samples. Further comparative studies will indicate whether more extensive decarboxylation has accompanied or has followed the deamination reactions implied in this study.

There are some interesting comparisons that can be made with earlier studies of amino acids in calcium carbonate systems (Hare, 1969; Wehmiller and Hare, 1971). Threonine and serine apparently behave quite differently. In the carbonate environment threonine is somewhat more 
TABLE 1

Ninhydrin Positive Compounds from Cariaco Trench Samples ${ }^{\text {a }}$

\begin{tabular}{l|rrr}
\hline \multirow{2}{*}{\multicolumn{1}{c|}{ Amino Acid }} & \multicolumn{3}{c}{ Sample Depth } \\
\cline { 2 - 4 } \multicolumn{1}{c}{$\begin{array}{c}1 \\
(\mathrm{~m})\end{array}$} & $\begin{array}{c}80 \\
(\mathrm{~m})\end{array}$ & $\begin{array}{r}110 \\
(\mathrm{~m})\end{array}$ \\
\hline Aspartic acid & 435 & 110 & 40 \\
Threonine & 125 & 30 & 20 \\
Serine & 95 & 30 & 25 \\
Glutamic acid & 255 & 70 & 40 \\
Proline & 250 & 35 & 20 \\
Glycine & 390 & 140 & 80 \\
Alanine & 310 & 120 & 75 \\
Valine & 260 & 110 & 75 \\
Methionine & 15 & 8 & 6 \\
Alloisoleucine & 8 & 5 & 6 \\
Isoleucine & 160 & 80 & 60 \\
Leucine & 250 & 110 & 90 \\
Tyrosine & 45 & 15 & 5 \\
Phenylalanine & 150 & 80 & 60 \\
$\beta$-Alanine & 150 & 160 & 170 \\
$\alpha$-Amino butyric acid & 80 & 80 & 60 \\
Histidine & 20 & 10 & 10 \\
Ornithine & 55 & 35 & 20 \\
Lysine & 270 & 125 & 75 \\
Arginine & 105 & 30 & 15 \\
Amino sugars & 780 & 460 & 450 \\
Ammonia & 6600 & 9300 & 9400 \\
Total micromoles/ & & & \\
gram & 10.8 & 11.1 & 10.8 \\
\hline & & &
\end{tabular}

${ }^{\mathrm{a}}$ Values expressed in nanomoles $\left(10^{-9}\right.$ moles $)$ per gram of freeze-dried sediment. stable than serine, while the data in this report seem to indicate that serine is more stable than threonine. Perhaps the presence of clays changes the pattern of diagenesis. The racemization of amino acids as evidenced by the appearance of alloisoleucine does not seem to be as easily interpreted in a heterogeneous sediment as it does in the carbonate environment. There seems to be little difference in the ratio of alloisoleucine to isoleucine at the 1-meter and 80 -meter levels. In a general way the ratio does increase with depth but the relationship does not appear to be consistent. Further work is in progress in studying the racemization reactions of other amino acids.

The total concentration of amino acids, exclusive of ammonia and amino sugars, is somewhat less than that reported by Degens (1965) for reducing sediments. Further studies should indicate the distribution pattern of amino acids associated with various phases within the sediment.

\section{REFERENCES}

Degens, E. T., 1965. Geochemistry of Sediments, Prentice-Hall Inc., Englewood Cliffs, N. J.

Hare, P. E., 1969. Geochemistry of proteins, peptides, and amino acids. In Organic Geochemistry, Methods and Results, G. Eglinton and M. T. J. Murphy, eds., Springer-Verlag, New York. 438.

1971. Ultrasensitive amino acid analyzer, Carnegie Institution of Washington Yearbook 70, 168. Wehmiller, J. and Hare, P. E., 1971. Racemization of amino acids in marine sediments. Science. 173, 907. 